\title{
BMJ Open Learning from positively deviant wards to improve patient safety: an observational study protocol
}

\author{
Ruth Baxter, ${ }^{1,2}$ Natalie Taylor, ${ }^{2,3}$ Ian Kellar, ${ }^{1,2}$ Rebecca Lawton ${ }^{1,2}$
}

To cite: Baxter R, Taylor N, Kellar I, et al. Learning from positively deviant wards to improve patient safety: an observational study protocol. BMJ Open 2015;5: e009650. doi:10.1136/ bmjopen-2015-009650

- Prepublication history and additional material is available. To view please visit the journal (http://dx.doi.org/ 10.1136/bmjopen-2015009650)

Received 6 August 2015 Revised 6 October 2015 Accepted 8 October 2015

\section{(1) crossark}

${ }^{1}$ School of Psychology, University of Leeds, Leeds, UK

${ }^{2}$ Quality and Safety Research Group, Bradford Institute for Health Research, Bradford, UK

${ }^{3}$ Centre for Healthcare Resilience and Implementation Science, Australian Institute of Health Innovation, Macquarie University, Sydney, New South Wales, Australia

Correspondence to Ruth Baxter; ps12rmb@leeds.ac.uk

\section{ABSTRACT}

Introduction: Positive deviance is an asset-based approach to improvement which has recently been adopted to improve quality and safety within healthcare. The approach assumes that solutions to problems already exist within communities. Certain groups or individuals identify these solutions and succeed despite having the same resources as others. Within healthcare, positive deviance has previously been applied at individual or organisational levels to improve specific clinical outcomes or processes of care. This study explores whether the positive deviance approach can be applied to multidisciplinary ward teams to address the broad issue of patient safety among elderly patients.

Methods and analysis: Preliminary work analysed National Health Service (NHS) Safety Thermometer data from 34 elderly medical wards to identify 5 'positively deviant' and 5 matched 'comparison' wards.

Researchers are blinded to ward status. This protocol describes a multimethod, observational study which will (1) assess the concurrent validity of identifying positively deviant elderly medical wards using NHS Safety Thermometer data and (2) generate hypotheses about how positively deviant wards succeed. Patient and staff perceptions of safety will be assessed on each ward using validated surveys. Correlation and ranking analyses will explore whether this survey data aligns with the routinely collected NHS Safety Thermometer data. Staff focus groups and researcher fieldwork diaries will be completed and qualitative thematic content analysis will be used to generate hypotheses about the strategies, behaviours, team cultures and dynamics that facilitate the delivery of safe patient care.

The acceptability and sustainability of strategies identified will also be explored.

Ethics and dissemination: The South East Scotland Research Ethics Committee 01 approved this study (reference: 14/SS/1085) and NHS Permissions were granted from all trusts. Findings will be published in peer-reviewed, scientific journals, and presented at academic conferences.

Trial registration number: This study is registered on the UK Clinical Research Network Study Portfolio (reference number-18050).

\section{INTRODUCTION}

Within healthcare, safety is currently defined as the absence of harmful incidents or

\section{Strengths and limitations of this study}

- This is the first known study to apply the positive deviance approach within the UK's National Health Service (NHS).

- Triangulating routinely collected safety data with staff and patient perceptions of safety will facilitate assessment of whether positively deviant wards have been correctly identified (concurrent validity).

- A theoretically underpinned framework will be used to guide qualitative data collection.

- The study will be conducted within one region of the UK and so quantitative analyses are limited in power and the positively deviant elderly medical wards identified may not demonstrate exceptional performance on a national scale.

events. ${ }^{1}{ }^{2}$ Healthcare organisations therefore focus on identifying the causes of these events and eliminating them. This reactive, deficit-based approach, commonly known as Safety I, does not typically explain why and how safe patient care is delivered. ${ }^{12}$ An alternative approach, known as Safety II, proposes that healthcare organisations should also focus on ensuring that 'as many things as possible go right'. ${ }^{2}$ It is argued that safe care is delivered routinely because clinicians continually adjust their behaviours to the different situations they face. Based on this approach, human factors are considered essential in providing flexibility and resilience rather than being potential sources of error. $^{2}$

Traditionally, methods used to improve patient safety address Safety I. For example, incident reporting, clinical auditing and quality improvement approaches such Statistical Process Control all identify, and aim to resolve, 'defects' or unacceptable variation in processes. ${ }^{3}{ }^{4}$ Despite extensive efforts to improve, there is little evidence that patient care is becoming any safer. ${ }^{5}{ }^{6} \mathrm{In}$ contrast, asset based approaches draw on strengths and resources which exist within 
communities. 'Positive deviance' adheres to the principles of Safety II and provides an asset-based approach to quality improvement. The approach is increasingly being used within healthcare organisations to improve quality and safety outcomes however limited guidance and evidence exists to support its application. ${ }^{7}$

\section{Positive deviance approach}

The positive deviance approach originated within international public health literature ${ }^{8}$ and has been used to address a number of intractable problems such as female genital mutilation and infection avoidance in drug users. ${ }^{9} 10$ Most famous positive deviance was used in Vietnam during the 1990s to sustain a $74 \%$ reduction in severe childhood malnutrition over 3 years. ${ }^{11} 12$

The positive deviance approach identifies and learns from those who demonstrate exceptional performance. It is built on the premise that solutions to enduring problems already exist within communities. Positively deviant individuals or groups are assumed to demonstrate uncommon behaviours and strategies which enable them to overcome problems and succeed. They do so despite facing the same constraints as others in the community. ${ }^{11} 1314$

Bradley et $a l^{14}$ propose a four-stage process to implement the approach within healthcare organisations (figure 1). Positively deviant individuals or groups are identified using routinely collected and validated data (stage 1). Qualitative methods are used to generate hypotheses about how these positive deviants succeed (stage 2). The hypotheses are tested in larger, representative samples to assess whether they improve the desired outcome (stage 3). Finally, the positively deviant behaviours are disseminated (stage 4 ).

Positive deviance can be distinguished from alternative quality improvement approaches in a number of ways. Its 'bottom up' philosophy ensures staff and patient involvement is integral throughout the process and, as a result, solutions to problems are internally driven rather than externally imposed. Positively deviant behaviours and strategies already facilitate exceptional performance, therefore, they should be feasible and sustainable within current resources, and acceptable to others. Consequently the positive deviance approach has potential to address some of the challenges faced within quality improvement projects such as convincing staff of the problem and that the chosen solution is effective, reducing unintended consequences, and sustaining results over time. ${ }^{15}$

Despite Bradley $e t a l \mathrm{~s}^{14}$ four-stage process, the quality of positive deviance studies within healthcare organisations is poor and limited guidance exists on how to conduct each stage ${ }^{13}$ While previous healthcare applications focus on specific outcomes or processes of care, ${ }^{7}$ such as reducing healthcare associated infections ${ }^{16} \quad 17$ and increasing guideline adherence for the treatment of acute myocardial infarction, ${ }^{14}$ few studies look more broadly at a range of safety issues. Positive deviants also tend to be identified at individual and organisational levels. ${ }^{7}$ Although safety is influenced at these levels, ${ }^{18}$ multidisciplinary ward teams are well-recognised microsystems, or clinical units, with their own processes, outcomes and cultures. ${ }^{19}$ If we are able to identify positively deviant wards that demonstrate success across a range of safety indicators, then we may be able to understand the latent or underlying factors associated with those teams.

\section{Aim, objectives and research questions}

To the best of our knowledge the positive deviance approach is yet to be applied within the UK's National Health Service (NHS). The approach is also rarely applied at a ward level to address broad issues such as patient safety. ${ }^{7}$ This observational study addresses the first two stages of the positive deviance process ${ }^{14}$ in order to (1) assess the concurrent validity of identifying positively deviant wards using routinely collected safety data and (2) generate hypotheses about how positively deviant wards deliver exceptionally safe patient care. Guidance to support the implementation of the positive deviance approach within healthcare organisations will also be generated.
Figure 1 The positive deviance process for healthcare organisations (adapted from Bradley et $a l^{14}$ ).



Baxter R, et al. BMJ Open 2015;5:e009650. doi:10.1136/bmjopen-2015-009650 
Elderly medical wards will be the focus of this study as these patients are particularly vulnerable to safety incidents. ${ }^{20}{ }^{21}$ We endeavour to identify positively deviant multidisciplinary ward teams who deliver safe patient care under particularly challenging circumstances.

Routinely collected and valid measures should be used to identify positive deviants. ${ }^{14}$ Although many routine measures of safety exist, few are available at ward level (eg, mortality statistics and the NHS staff survey ${ }^{22}{ }^{23}$ ). The NHS Safety Thermometer (ST) is published on the Health and Social Care Information Centre (HSCIC) at trust (organisation), specialty and ward level. ${ }^{24}$ Data are collected monthly on all acute wards for four common patient harms: falls, pressure ulcers, venous thromboembolism (VTEs) and urinary infections in catheterised patients (UTIs). These are combined to create a composite measure of 'harm-free care'. While concerns exist about the reliability and validity of ST data, ${ }^{25}$ this is the only routinely collected measure of overall safety, available at ward level, from all NHS trusts. Furthermore the measures included are particularly pertinent to our elderly patient population.

The following primary research questions will be addressed:

1. Can NHS ST data be used for the valid and reliable identification of positively deviant elderly medical wards?

2. What strategies and behaviours do multidisciplinary teams use to deliver exceptionally safe patient care on elderly medical wards?

3. How do team dynamics and culture differ between elderly medical wards that deliver exceptionally safe and averagely safe patient care?

The following secondary research question will be addressed:

1. To what extent do organisational, situational and individual factors help or hinder the delivery of safe patient care on exceptional and averagely performing elderly medical wards?

Prior to addressing these research questions, preliminary work outlined below was conducted to identify a sample of positively deviant and comparison elderly medical wards with exceptional (potentially positively deviant) and slightly-above-average safety performances. Results of this analysis will be reported fully in a separate publication.

\section{PRELIMINARY WORK: IDENTIFYING POSITIVELY DEVIANT WARDS}

This study is being conducted in a region of northern England containing 13 acute NHS trusts. Clinical leads in each trust were contacted to identify, and provide basic information about, each of their elderly medical wards (bed numbers, patient gender and approximate patient age). Thirty-seven wards were identified across the region, all of which fulfilled the inclusion criteria in box 1 .

ST data were extracted at ward and trust level from the HSCIC for the period August 2013 to July 2014 (the
Box 1 Inclusion criteria for 'elderly medical' wards

Dedicated care for patients over the age of 65 years

- Provision of $24 \mathrm{~h}$, acute, medical care

- Typical patient stay exceeds $48 \mathrm{~h}$ (excluding assessment units)

- Dedicated medical care (excluding specialty wards, eg, stroke or rehabilitation)

Dedicated multidisciplinary ward team

most recent 12 months). The trust level data sets accounted for patients being over the age of 70 years and cared for in acute settings. Data were available for 36 wards and 13 trusts. Two wards, with $<6$ months of data, were excluded.

Cross-sectional and temporal analyses were conducted to identify positively deviant elderly medical wards with exceptional safety performances. For the 12-month period an average performance for 'harm-free care' was calculated and wards were ranked to identify the 'best' within the region. Given that wards are the unit of analysis, it was necessary to limit the extent to which organisational and specialty/directorate level factors facilitate safety. A scatterplot therefore compared ward and trust level data to ensure ward performance was not just a function of their respective trusts' exceptional safety record.

To assess performance over time run charts compared the monthly performance of each ward with the average monthly performance across the region. Run charts were visually assessed to identify wards that consistently outperformed the regional average over the 12-month period.

Wards with slightly above-average harm-free care performance were selected as a comparison group. Our aim was to explore how positive deviants excel from the majority of the population (from the average) rather than to explore how they differ from those who perform poorly. Comparison wards were matched to the positively deviant wards using three variables: trust status, patient gender and a measure of deprivation, to ensure that safe patient care was not purely a function of caring for affluent populations (Index of Multiple Deprivation ${ }^{26}$ ). Five positively deviant and five matched comparison wards were identified and invited to participate in the study. One ward was unable to participate therefore the final sample includes nine wards.

\section{PRIMARY DATA COLLECTION: METHODS AND ANALYSES Study design and setting}

The preliminary work above identified a sample of 'positively deviant' and 'comparison' elderly medical wards based on ST performance. RB (the primary researcher) and staff in our participating wards will be blind to whether the wards are in the positively deviant or comparison groups throughout the course of the study described below.

An observational, multimethods study will be conducted on nine elderly medical wards. It will be 
conducted in two simultaneous phases. During the quantitative phase data will be collected using validated staff and patient surveys to provide two different perspectives of safety on each ward. These data will be analysed alongside the ST data to explore whether the ST has concurrent validity, that is, whether it correlates with the other validated measures (research question 1). The qualitative phase explores how positively deviant wards deliver exceptionally safe patient care. Multidisciplinary team focus groups will be conducted and researchers will keep fieldwork diaries to capture the strategies, behaviours, team dynamics and cultures that facilitate delivery of safe patient care (research questions 2 and 3). Data from the staff surveys will be used to assess how organisational, situational and individual factors influence the delivery of safe care (research question 4).

\section{QUANTITATIVE PHASE: ASSESSING THE ST'S CONCURRENT VALIDITY}

\section{Participants: eligibility and recruitment}

Patients

Patients who have capacity and are physically well enough will be invited to participate in a survey. They must be over 65 years of age and have received care on the ward for more than $4 \mathrm{~h}$. Opportunity sampling will be used to recruit up to 20 patients per ward and eligibility will be determined by ward sisters and/or nurses on shift. Researchers will discuss the study verbally, provide patients with a written information sheet, and give them the opportunity to consider the information and ask questions. Participating patients must provide informed consent.

\section{Multidisciplinary staff}

Multidisciplinary ward staff, from all job roles and professional grades, will be invited to participate in the staff surveys. Opportunity sampling will be used to recruit up to $50 \%$ of the team per ward. Staff will be provided with a letter about the research and posters will be displayed in staff areas on the ward.

\section{Data collection: measures, tools and procedures}

The majority of data collection will be undertaken by $\mathrm{RB}$, with support from two additional researchers (CR and $\mathrm{AH}$ ).

\section{Patient surveys}

Patients will complete the Patient Measure of Safety (PMOS) which gathers feedback from hospitalised patients about the safety of their care and assesses perceptions about factors contributing to safety. ${ }^{27}$ The survey (see online additional file 1) includes 44 items measuring nine domains: communication and team working, organisation and care planning, access to resources, ward type and layout, information flow, staff roles and responsibilities, staff training, equipment (design and functioning), and delays. Two stand-alone items measure 'dignity and respect' and 'The Friends and Family Test' (FFT; a measure of patient experience used nationally within the $\mathrm{UK}^{28}$ ). Patients respond to each question using five-point Likert scale ranging from 'strongly disagree' to 'strongly agree'. 'Not applicable' and 'prefer not to answer' options are available and comments can be added to each answer to provide context. ${ }^{29}$ The PMOS is valid, reliable and acceptable to patients. ${ }^{27} 29$

Depending on their preference patients will complete the survey either with the researcher's support (the researcher will read the questions and record their answers) or independently. This flexibility will help researchers overcome some of the challenges associated with collecting data within elderly populations. ${ }^{30} 31$ Surveys are expected to take approximately $20 \mathrm{~min}$ and can be completed electronically or using paper and pen. A 'thank you' card will be given on completion.

\section{Staff surveys}

Staff will complete the Patient Safety Grade (PSG) which is one of four outcomes within the Hospital Survey on Patient Safety Culture (HSOPSC). ${ }^{32}$ The single item question asks staff to grade their ward on overall patient safety using a five-point Likert scale ranging from 'excellent' to 'failing'. The HSOPSC has been extensively validated and guidance suggests that outcomes which are not required can be removed ${ }^{32-34}$ In a recent study, the PMOS and the HSOPSC demonstrated strong correlations with the ST measure of 'harm-free care'. ${ }^{35}$ Of all HSOPSC outcomes the PSG correlated most strongly.

Multidisciplinary staff will receive a letter informing them about the study. This letter will enclose a copy of the survey, an information sheet and return envelope. Participating staff will place completed surveys within a secure 'drop box' on the ward. The survey takes approximately $10 \mathrm{~min}$ and will be incentivised by a $£ 20$ prize draw on each ward. Additional survey content is described under the Yorkshire Contributory Factors framework heading, and the full survey can be viewed in online additional file 2 .

\section{NHS ST}

Data collection will start several months after the identification of elderly medical wards due to the time delay imposed by the process of gaining NHS ethical approvals. Ward-level ST data will therefore be extracted from the HSCIC for the same time period as primary data collection to explore whether wards retain their exceptional or slightly above-average performance levels.

\section{Average patient age data}

During preliminary work accurate average patient age data was not available for all 36 wards. To exclude the possibility that positively deviant wards provide safer care because they treat a comparatively younger group of patients, average patient age data for the annual period 
of 1 August 2013 to 31 July 2014 will be collected from each ward.

\section{Quantitative analysis}

Blinding will be removed prior to analysis. Descriptive statistics will summarise the data and assess whether the assumptions of parametric tests are fulfilled. All individual data will be aggregated to ward level for analyses. PMOS items will be averaged to create scores for the overall survey and each domain. Average ward level scores for the PSG will also be calculated.

PMOS, PSG and ST data (for both time periods) will be correlated to assess the concurrent validity of the ST data; whether the ST can confidently be used as a measure for identifying positively deviant elderly medical wards. Scatter plots will explore whether relationships between variables are linear. Wards will also be ranked to assess whether positively deviant wards generally perform better than comparison wards across all measures. An independent samples t test will explore whether average patient age significantly differs between the two groups.

\section{QUALITATIVE PHASE: EXPLORING HOW POSITIVE DEVIANTS SUCCEED}

Participants: eligibility and recruitment

Multidisciplinary ward staff from all roles and professional grades will be invited to participate in focus groups. Opportunity and purposive sampling will be used to recruit approximately eight members of staff on each ward and recruitment will be supported by ward sisters. Staff will provide written informed consent to participate in focus groups.

\section{Data collection: measures, tools and procedures \\ Staff focus groups}

One focus group, lasting up to $60 \mathrm{~min}$, will be conducted on each ward. Suitable times and locations will be arranged with ward sisters, and staff will be given written and verbal explanations of the study. Following an opportunity to ask questions written informed consent will be gained.

Simply asking staff to discuss how they deliver 'safe care' may not lead to in-depth conversations, therefore, an adapted version of the Manchester Patient Safety Framework (MaPSaF) will be used to help structure focus group discussions. The MaPSaF is a tool to qualitatively explore safety culture within the $\mathrm{NHS}^{36}$ which is theoretically underpinned by Westrum's Model of Organisational Development. ${ }^{37} 38$ Following advice from the developer, Dianne Parker, this tool was adapted and shortened to contain five dimensions of safety culture: commitment to overall continuous improvement; priority given to safety; recording, evaluating and learning from incidents and best practice; communication about safety issues; and team-working (see online additional file 3).
Staff will be given approximately $10 \mathrm{~min}$ to read the framework and rate their ward on each domain. They will identify which domains they think their ward particularly excels at. The primary researcher (RB) will use a semistructured discussion guide, based on the MaPSaF (see online additional file 4), to facilitate further discussion about the domains they identify. The discussion will aim to help staff identify the specific strategies and behaviours they use to successfully deliver safe patient care. Focus groups will be audio recorded. Refreshments and a $£ 30$ gift voucher will incentivise attendance.

\section{Researcher fieldwork diaries}

Fieldwork diaries will be completed by the chief investigator (RB) following ward visits and interactions with staff. Guidance for fieldwork diaries will be used to promote consistent observations across wards (see online additional file 5). Where possible, five recognised behavioural constructs of team performance will be observed: communication, coordination, cooperation and backup, leadership, and monitoring and situational awareness. $^{39}$ Researchers will also observe staff and patient interactions, staffing levels and workload, patient case mix, and ward engagement with the research.

\section{Yorkshire Contributory Factors framework}

The Yorkshire Contributory Factors framework (YCF) contains 19 organisational, situational and individual factors known to contribute to patient safety incidents. ${ }^{18}$ Questions addressing each of these factors have been included in the quantitative staff survey (see online additional file 2). Staff will rate the extent to which each factor helps or hinders the delivery of safe care using five-point Likert scale ranging from 'extremely helps' to 'extremely hinders'.

\section{Feedback meetings}

Following analysis, informal feedback meetings will be scheduled on each ward to discuss the positively deviant strategies identified. Researchers will gain feedback from staff about their validity, acceptability and sustainability.

\section{Qualitative analysis}

Audio recordings of focus groups will be transcribed verbatim, anonymised, and then analysed using qualitative thematic content analysis. ${ }^{40}{ }^{41}$ Content analysis has recently evolved to include interpretations of latent content in addition to the traditional quantitative descriptions of data. It can be conducted to various levels of abstraction by focusing on the manifest (explicit data) or latent content (abstract data requiring deeper interpretation). ${ }^{40}{ }^{41}$ This study aims to (1) identify the concrete strategies and behaviours used to deliver safe patient care and (2) identify the abstract factors that facilitate success such as team culture and dynamics. Qualitative thematic content analysis facilitates interpretation at these two different levels. 
Qualitative thematic content analysis is conducted in three stages. ${ }^{41}$ 'Preparation' involves immersion in the data. During the 'organising' stage data is analysed to create codes which are combined to form higher order categories and subcategories. The final 'reporting' stage refers to the presentation of analyses and results.

To ensure rigour, decisions made during the analysis will be documented in a reflexive diary. A proportion of transcripts will be independently analysed to assess intercoder reliability, and researchers will meet regularly to discuss and resolve coding problems.

\section{ETHICS AND DISSEMINATION}

The following permissions have been obtained for this study:

- NHS Ethics-approval granted by the South East Scotland Research Ethics Committee 01 (reference: 14/SS/1085);

- NHS Permissions were granted by all trusts involved;

- This study is registered on the UK Clinical Research Network Study Portfolio (reference-18050).

Informed consent will be gained from patients and all staff attending focus groups. Informed consent will be assumed for staff who return surveys. Confidentiality and anonymity will be strictly maintained. Data will be anonymised and aggregated to ward level and participants will not be identified through any data, transcripts, or publications.

During the planning phase, stakeholder meetings were held with patient and staff representatives, both of whom perceived the word 'deviance' negatively. More positive terminology, such as successful and/or exceptional wards, will therefore be used instead of the term 'positive deviance'.

This study forms part of the primary researcher's $\mathrm{PhD}$ whose thesis will be assessed by the University of Leeds. Findings will be shared with the wards involved and disseminated widely through peer reviewed, scientific journals, and at national and international conferences.

\section{STUDY STATUS}

Data collection started in February 2015 and is expected to last 5-6 months. Feedback meetings will be scheduled between September and October 2015.

\section{DISCUSSION}

This protocol extends current literature to assess positive deviance at a ward level in relation to the broad, complex problem of patient safety. It is the first known application of positive deviance within a NHS setting. The majority of previous studies within healthcare have been conducted in the USA to address specific outcomes or processes of care, at individual or organisational levels. ${ }^{7}$ Although further research is required to test hypotheses before disseminating them more widely (stages 3 and 4 of the positive deviance process), we expect the wards involved will benefit and learn from each other to improve safety even further.

More broadly, we aim to develop additional guidance to help others implement positive deviance within healthcare settings. Our findings will clarify: what routinely collected data can be used to identify positively deviant wards and how it can be analysed; the timescale positive deviants can be identified over; and the methods that can be used explore how they succeed.

While planning this study we have grappled with fundamental questions surrounding the approach. These include: What constitutes positively deviant behaviour? How is positive deviance different to high performance? How confident must we be that positive deviants have been correctly identified? Who should positive deviants be compared to? It is essential that studies such as this are conducted to address these fundamental questions. Until we can evaluate the effectiveness of the approach within healthcare organisations, we are unable to conclude whether positive deviance is an improvement method worth investing in.

\section{Twitter Follow Ruth Baxter at @RuthMBaxter}

Acknowledgements The authors would like to thank Professor Mohammed Mohammed and Ms Victoria Pye who supported the preliminary work to identify a sample of positively deviant and comparison wards. The authors would also like to recognise the following people for their support designing, setting up and conducting this research: Professor Diane Parker, Ms Caroline Reynolds, Ms Alex Howat and Ms Liz Thorp.

Collaborators RB, IK and RL are part of the Evidence Based Transformation Theme of the NIHR CLAHRC Yorkshire and Humber. This paper presents independent research by the National Institute for Health Research Collaboration for Leadership in Applied Health Research and Care Yorkshire and Humber (NIHR CLAHRC YH). http://www.clahrc-yh.nir.ac.uk.

Disclaimer The views and opinions expressed are those of the authors, and not necessarily those of the NHS, the NIHR or the Department of Health.

Contributors RB, NT, IK and RL substantially contributed to the conception and design of this study. All authors critically assessed and approved the final study design. RB was primarily responsible for setting up the study, gaining ethical approvals and starting data collection. RB drafted the initial manuscript. NT, IK and RL assessed the manuscript critically for intellectual content. All authors approved the final manuscript.

Funding The Health Foundation funded the PhD studentship through which this study is being completed.

\section{Competing interests None declared.}

Ethics approval NHS Ethics-South East Scotland Research Ethics Committee 01.

Provenance and peer review Not commissioned; externally peer reviewed.

Open Access This is an Open Access article distributed in accordance with the Creative Commons Attribution Non Commercial (CC BY-NC 4.0) license, which permits others to distribute, remix, adapt, build upon this work noncommercially, and license their derivative works on different terms, provided the original work is properly cited and the use is non-commercial. See: http:// creativecommons.org/licenses/by-nc/4.0/

\section{REFERENCES}

1. Hollnagel E, Braithwaite J, Wears RL. Resilient health care. UK: Ashgate, 2013. 
2. Hollnagel E, Leonhardt J, Licu T, et al. From Safety-I to Safety-II: a white paper. Brussels: European Organisation for the Safety of Air Navigation (EUROCONTROL), 2013.

3. Power M, Stewart K, Brotherton A. What is the NHS Safety Thermometer? Clin Risk 2012;18:163-9.

4. The Health Foundation. Quality improvement made simple. London, UK: The Health Foundation, 2013.

5. Vincent C, Aylin P, Franklin BD, et al. Is health care getting safer? BMJ 2008;337:a2426.

6. Landrigan $\mathrm{CP}$, Parry GJ, Bones $\mathrm{CB}$, et al. Temporal trends in rates of patient harm resulting from medical care. $N$ Engl $J$ Med 2010;363:2124-34

7. Baxter R, Kellar I, Taylor N, et al. How is the positive deviance approach applied within healthcare organizations? A systematic review of methods used. BMC Health Services Research: Evidence-based practice; 1-3 July 2014; UK, London: BMC Health Services Research, 2014:7.

8. Wishik SM, Van Der Vynckt S. The use of nutritional 'Positive Deviants' to identify approaches for modification of dietary practices. Am J Public Health 1976;66:38-42.

9. Friedman SR, Mateu-Gelabert P, Sandoval M, et al. Positive deviance control-case life history: a method to develop grounded hypotheses about successful long-term avoidance of infection. BMC Public Health 2008;8:94.

10. Masterson JM, Swanson JH. Female genital cutting: breaking the silence enabling change. Washington DC: International Center for Research on Women (ICWR) and the Center for Development and Population Activities (CEDPA), 2000.

11. Marsh DR, Schroeder DG, Dearden KA, et al. The power of positive deviance. BMJ 2004;329:1177-9.

12. Mackintosh UAT, Marsh DR, Schroeder DG. Sustained positive deviant child care practices and their effects on child growth in Vietnam. Food Nutr Bull 2002;23(4 Suppl):18-27.

13. Lawton R, Taylor N, Clay-Williams R, et al. Positive deviance: a different approach to achieving patient safety. BMJ Qual Saf 2014;23:880-3.

14. Bradley EH, Curry LA, Ramanadhan S, et al. Research in action: using positive deviance to improve quality of health care. Implement Sci 2009;4:25.

15. Dixon-Woods M, McNicol S, Martin G. Ten challenges in improving quality in healthcare: lessons from the Health Foundation's programme evaluations and relevant literature. BMJ Qual Saf 2012;21:876-84.

16. Marra AR, Guastelli LR, De Araujo CMP, Jr, et al. Positive deviance: a new strategy for improving hand hygiene compliance. Infect Control Hosp Epidemiol 2010;31:12-20.

17. Marra AR, Guastelli LR, Pereira de Araujo CM, et al. Positive deviance: a program for sustained improvement in hand hygiene compliance. Am J Infect Control 2011;39:1-5.

18. Lawton R, McEachan RR, Giles SJ, et al. Development of an evidence-based framework of factors contributing to patient safety incidents in hospital settings: a systematic review. BMJ Qual Saf 2012;21:369-80.

19. Nelson EC, Batalden PB, Huber TP, et al. Microsystems in health care: Part 1. Learning from high-performing front-line clinical units. Jt Comm J Qual Improv 2002;28:472-93.

20. Sari AB, Cracknell A, Sheldon TA. Incidence, preventability and consequences of adverse events in older people: results of a retrospective case-note review. Age Ageing 2008;37:265-9.
21. Thomas EJ, Brennan TA. Incidence and types of preventable adverse events in elderly patients: population based review of medical records. BMJ 2000;320:741-4.

22. Health and Social Care Information Centre. Summary Hospital-level Mortality Indicator. 2015. http://www.hscic.gov.uk/SHMI (accessed 31 Jul 2015).

23. Picker Institute Europe. NHS Staff Survey. 2015. http://www. nhsstaffsurveys.com/Page/1010/Home/Staff-Survey-2014/ (accessed 31 Jul 2015).

24. Health and Social Care Information Centre. NHS Safety Thermometer. 2014. http://www.hscic.gov.uk/thermometer (accessed 29 Apr 2014).

25. Power M, Fogarty M, Madsen J, et al. Learning from the design and development of the NHS Safety Thermometer. Int J Qual Health Care 2014;26:287-97.

26. Office of the Deputy Prime Minister. The English indices of deprivation 2004: summary (revised). UK: Office of the Deputy Prime Minister, 2004

27. Giles SJ, Lawton RJ, Din I, et al. Developing a patient measure of safety (PMOS). BMJ Qual Saf 2013;22:554-62.

28. NHS England. Friends and family test. 2014. http://www.england nhs.uk/statistics/statistical-work-areas/friends-and-family-test/ (accessed 29 Apr 2014).

29. McEachan RRC, Lawton RJ, O'Hara JK, et al. Developing a reliable and valid patient measure of safety in hospitals (PMOS): a validation study. BMJ Qual Saf 2014;23:565-73.

30. Uman GC, Urman HN. The challenges of conducting clinical nursing research with elderly populations. AORN J 1990;52:400-6.

31. Hall S, Longhurst S, Higginson IJ. Challenges to conducting research with older people living in nursing homes. BMC Geriatr 2009;9:38

32. Sorra J, Nieva V. Hospital survey on patient safety culture. Rockville: AHRQ Publication No. 04-00412004.

33. Sarac C, Flin R, Mearns K, et al. Hospital survey on patient safety culture: psychometric analysis on a Scottish sample. BMJ Qual Saf 2011;20:842-8

34. Sorra J, Dyer N. Multilevel psychometric properties of the AHRQ hospital survey on patient safety culture. BMC Health Serv Res 2010;10:199.

35. Lawton R, O'Hara JK, Sheard L, et al. Can staff and patient perspectives on hospital safety predict harm-free care? An analysis of staff and patient survey data and routinely collected outcomes. BMJ Qual Saf 2015;24:369-76.

36. National Patient Safety Agency. Manchester Patient Safety Framework (MaPSaF). 2006. http://www.nrls.npsa.nhs.uk/resources/ ?entryid45=59796 (accessed 7 Aug 2014).

37. Parker D. Managing risk in healthcare: understanding your safety culture using the Manchester Patient Safety Framework (MaPSaF). J Nurs Manag 2009;17:218-22.

38. Westrum R. A typology of organisational cultures. Qual Saf Health Care 2004;13(Suppl_2):ii22-7.

39. Undre S, Healey AN, Darzi A, et al. Observational assessment of surgical teamwork: a feasibility study. World J Surg 2006;30:1774-83.

40. Graneheim UH, Lundman B. Qualitative content analysis in nursing research: concepts, procedures and measures to achieve trustworthiness. Nurse Educ Today 2004:24:105-12.

41. Vaismoradi $\mathrm{M}$, Turunen $\mathrm{H}$, Bondas $\mathrm{T}$. Content analysis and thematic analysis: implications for conducting a qualitative descriptive study. Nurs Health Sci 2013;15:398-405. 